\title{
Excedance Numbers for the Permutations of Type $B$
}

\author{
Alina F.Y. Zhao \\ School of Mathematical Sciences and Institute of Mathematics \\ Nanjing Normal University, Nanjing 210023, P.R. China \\ alinazhao@njnu.edu.cn
}

Submitted: May 21, 2012; Accepted: May 8, 2013; Published: May 16, 2013

Mathematics Subject Classifications: 05A05, 05A19

\begin{abstract}
This work provides a study on the multidistribution of type $B$ excedances, fixed points and cycles on the permutations of type $B$. We derive the recurrences and closed formulas for the distribution of signed excedances on type $B$ permutations as well as derangements via combinatorial construction. Based on this result, we obtain the recurrence and generating function for the signed excedance polynomial and disclose some relationships with Euler numbers and Springer numbers, respectively.
\end{abstract}

Keywords: type $B$ permutation; type $B$ excedance; type $B$ derangement; fixed point; cycle; Euler number; Springer number

\section{Introduction}

Let $\mathfrak{S}_{n}$ be the set of permutations of $[n]:=\{1,2, \ldots, n\}$. A permutation $\pi$ of $[n]$ can be written in one-line notation as $\pi=\pi_{1} \pi_{2} \cdots \pi_{n}$, where $\pi_{i}=\pi(i), i=1,2, \ldots, n$; or as a disjoint union of its distinct cycles $C_{1}, \ldots, C_{k}$, i.e., $\pi=C_{1} \cdots C_{k}$. A cycle $C$ is said to be in standard form if its smallest element is in the first position. For each $\pi \in \mathfrak{S}_{n}$, the excedance set and the excedance number of $\pi=\pi_{1} \pi_{2} \cdots \pi_{n}$ are defined as

$$
\operatorname{Exc}(\pi)=\left\{i \in[n]: \pi_{i}>i\right\} \quad \text { and } \operatorname{exc}(\pi)=|\operatorname{Exc}(\pi)|, \text { respectively. }
$$

Let $B_{n}$ be the hyperoctahedral group on $[n]$, where each element in $B_{n}$ can be regarded as a signed permutation or a permutation of type $B$ on $[n]$; in other words, we view each $\pi \in B_{n}$ as a function $\pi:[n] \rightarrow[-n, n] \backslash\{0\}$ such that $|\pi| \in \mathfrak{S}_{n}$ and $|\pi|(i)=|\pi(i)|$ for $i \in[n]$. For $\pi=\pi_{1} \pi_{2} \cdots \pi_{n} \in B_{n}$, we denote by $\bar{i}$ the negative element $-i$, and use the natural order for the elements of $\pi$, i.e.,

$$
\bar{n}<\overline{n-1}<\cdots<\overline{2}<\overline{1}<1<2<\cdots<n-1<n .
$$


The cycle decomposition of $\pi \in B_{n}$ is accomplished by first writing $|\pi|$ as the disjoint union of cycles, and then placing bars on the elements which have bars in $\pi$. For example, let $\pi=\overline{3} 51 \overline{7} 29 \overline{6} \overline{8} \overline{4} \in B_{9}$, we have $|\pi|=351729684=(1,3)(2,5)(4,7,6,9)(8)$, and thus the cycle decomposition of $\pi$ is $(1, \overline{3})(2,5)(\overline{4}, \overline{7}, \overline{6}, 9)(\overline{8})$. The cycle of a type $B$ permutation is also called a signed cycle. Given a cycle $C$, denoted by $l(C)$ the length of $C$, that is the number of elements in the cycle $C$.

For a permutation $\pi=\pi_{1} \pi_{2} \cdots \pi_{n}$ in $\mathfrak{S}_{n}$ or $B_{n}$, let $\operatorname{cyc}(\pi)$ be the number of cycles in the cycle decomposition of $\pi$. A fixed point of $\pi$ is an element $i$ such that $\pi_{i}=i$, and further denote by fix $(\pi)$ the number of fixed points in $\pi$; this permutation is called a derangement if $\pi_{i} \neq i$ for all $i \in[n]$, i.e., a permutation without fixed points. In order to distinguish, a derangement in $B_{n}$ is referred as a type $B$ derangement. Denote by the set of derangements and the set of type $B$ derangements on $[n]$ by $D_{n}$ and $D_{n}^{B}$, respectively.

Let us recall the definition of type $B$ excedances introduced by Brenti [5].

Definition 1. For $\pi \in B_{n}$ and $i \in[n],\left|\pi_{i}\right|$ is said to be a type $B$ excedance of $\pi$ if $\pi_{i}=\bar{i}$ or $\pi_{\left|\pi_{i}\right|}>\pi_{i}$, and we denote by $\operatorname{exc}_{B}(\pi)$ the number of type $B$ excedances of $\pi$.

It is easy to determine the type $B$ excedance set of $\pi \in B_{n}$ from its cycle decomposition. That is, for a cycle $\left(c_{1}, c_{2}, \ldots, c_{m}\right)(m \geqslant 2)$ of $\pi,\left|c_{i}\right|$ is a type $B$ excedance if $c_{i}<$ $c_{i+1}(1 \leqslant i \leqslant m)$, where $c_{m+1}=c_{1}$; or $i$ is a type $B$ excedance if $(\bar{i})$ is a cycle of $\pi$. For example, for the type $B$ permutation $\pi=\overline{3} 51 \overline{7} 29 \overline{6} \overline{8} \overline{4}$ with cycle decomposition $(1, \overline{3})(2,5)(\overline{4}, \overline{7}, \overline{6}, 9)(\overline{8})$, we have $\operatorname{exc}_{B}(\pi)=5$ since $\overline{3}<1,2<5, \overline{7}<\overline{6}, \overline{6}<9$ and $\pi_{8}=\overline{8}$.

For $\pi \in \mathfrak{S}_{n}$, the (weighted) signed excedance of $\pi$ is defined as $(-1)^{\operatorname{cyc}(\pi)} x^{\operatorname{exc}(\pi)}$, and from the generating function point of view, Brenti [6] derived that

$$
\sum_{\pi \in \mathfrak{S}_{n}}(-1)^{\operatorname{cyc}(\pi)} x^{\operatorname{exc}(\pi)}=-(x-1)^{n-1} .
$$

Then, Ksavrelof and Zeng [12] presented a combinatorial proof by introducing the enumerative polynomial on $\mathfrak{S}_{n}$ as follows:

$$
P_{n}(x, y, z)=\sum_{\pi \in \mathfrak{S}_{n}} x^{\operatorname{exc}(\pi)} y^{\mathrm{fix}(\pi)} z^{\operatorname{cyc}(\pi)} .
$$

Bagno and Garber [4] generalized this result to colored permutation groups with other definitions of excedances, and later Bagno et al. [3] defined the excedance number for the multi-colored permutation group and calculated its multi-distribution with the number of fixed points and cycles. However, the study on type $B$ excedances remains almost untouched. In this paper, we will provide a study on the joint distribution of the number of type $B$ excedances, fixed points and cycles in the permutations of type $B$. We first give the following definition of the signed excedance polynomial $P_{n}^{B}(x, y, z)$.

Definition 2. The type $B$ signed excedance polynomials are given by

$$
P_{n}^{B}(x, y, z)=\sum_{\pi \in B_{n}} x^{\operatorname{exc}(\pi)} y^{\operatorname{fix}(\pi)} z^{\operatorname{cyc}(\pi)} \quad \text { for } n \geqslant 1,
$$

and we set $P_{0}^{B}(x, y, z)=1$ when $n=0$. 
It is easy to find that $P_{n}^{B}(x, 1,1)$ and $P_{n}^{B}(x, 0,1)$ correspond to the classical Eulerian polynomial of type $B([5,9])$ and the derangement polynomial of type $B([8,11])$, respectively.

This paper is organized as follows. We construct involutions on type $B$ permutations and type $B$ derangements to derive the recurrences and explicit formulas for $P_{n}^{B}(x, 1,-1)$ and $P_{n}^{B}(x, 0,-1)$ in Sections 2 and 3, respectively. Based on combinatorial arguments, we derive the recurrence relation and generating function for $P_{n}^{B}(x, y, z)$ in Section 4 , and further disclose some relationships between $P_{n}^{B}(-1,1,1)$ and the Euler number, as well as relationships between $P_{n}^{B}(-1,0,1)$ and the Springer number.

\section{Signed excedances on type $B$ permutations}

In this section, we will study the signed excedances on type $B$ permutations, and derive the recurrence and closed form for $P_{n}^{B}(x, 1,-1)$ as follows.

Theorem 3. For $n \geqslant 3$, we have

$$
\begin{aligned}
& P_{n}^{B}(x, 1,-1)=(x-1)^{2} P_{n-2}^{B}(x, 1,-1), \\
& P_{n}^{B}(x, 1,-1)= \begin{cases}-(x+1)(x-1)^{n-1}, & \text { if } n \text { is odd }, \\
(x-1)^{n}, & \text { if } n \text { is even },\end{cases}
\end{aligned}
$$

where $P_{1}^{B}(x, 1,-1)=-(x+1), P_{2}^{B}(x, 1,-1)=(x-1)^{2}$.

Our proof technique is based on the construction of sign-reversing involutions which preserve the number of excedances yet change the parity of the number of cycles of a type $B$ permutation. For our discussion, the weight of the type $B$ permutation $\pi \in B_{n}$ is defined as $w(\pi)=(-1)^{\operatorname{cyc}(\pi)} x^{\operatorname{exc}_{B}(\pi)}$, and the weight of a set is given by the sum over all weights of the elements in this set.

\subsection{Combinatorial proof of the recurrence (2)}

We first partition the set $B_{n}$ into four subsets as follows:

- $B_{n}^{1}:=\left\{\pi \in B_{n} \mid \pi_{n-1}=\overline{n-1}\right.$ and $\left.\pi_{n}=\bar{n}\right\}$;

- $B_{n}^{2}:=\left\{\pi \in B_{n} \mid \pi_{n-1}=n-1\right.$ and $\left.\pi_{n}=n\right\}$;

- $B_{n}^{3}:=\left\{\pi \in B_{n} \mid \pi_{n-1}=n\right.$ and $\left.\pi_{n}=n-1\right\} \bigcup\left\{\pi \in B_{n} \mid \pi_{n-1}=\bar{n}\right.$ and $\left.\pi_{n}=\overline{n-1}\right\}$;

- $B_{n}^{4}:=B_{n} \backslash\left\{B_{n}^{1} \cup B_{n}^{2} \cup B_{n}^{3}\right\}$.

It is obvious that the map $\pi \mapsto \pi^{\prime \prime}=\pi_{1} \pi_{2} \cdots \pi_{n-2}$ gives a bijection between set $B_{n}^{1}$ and set $B_{n-2}$ such that $w(\pi)=x^{2} w\left(\pi^{\prime \prime}\right)$. Therefore the weight of $B_{n}^{1}$ is $x^{2} P_{n-2}^{B}(x, 1,-1)$. Similarly, the weights of $B_{n}^{2}$ and $B_{n}^{3}$ are $P_{n-2}^{B}(x, 1,-1)$ and $-2 x P_{n-2}^{B}(x, 1,-1)$, respectively. Thus, it remains to prove that the weight of $B_{n}^{4}$ is equal to zero. 
For convenience, we call $\pi_{i}$ a singleton if $\pi_{i}=i$ or $\pi_{i}=\bar{i}$, i.e., $\pi_{i}$ is in a cycle of length exactly one. Now we construct a sign-reversing involution $\varphi$ on $B_{n}^{4}$ by

$$
\pi \mapsto \pi^{\prime}=\left(\pi_{n-1}, \pi_{n}\right) \circ \pi
$$

and it will be proven to be the desired involution by analyzing the number of type $B$ excedances and cycles between $\pi$ and $\pi^{\prime}$. Without loss of generality, assume that $\pi_{n-1}$ and $\pi_{n}$ lie in two different cycles $C_{1}$ and $C_{2}\left(C_{1} \neq C_{2}\right)$, respectively. We will complete the proof by considering the following cases according to the lengths of the cycles $C_{1}$ and $C_{2}$.

i) For $l\left(C_{1}\right)=l\left(C_{2}\right)=1$, we have $\pi=\cdots(n-1) \cdots(\bar{n}) \cdots$ or $\pi=\cdots(\overline{n-1}) \cdots(n) \cdots$ from $\pi \notin B_{n}^{1} \cup B_{n}^{2}$. For the former, it holds that $\pi^{\prime}=\cdots(n-1, \bar{n}) \cdots$, while for the latter, $\pi^{\prime}=\cdots(\overline{n-1}, n) \cdots$. This gives $w(\pi)=-w\left(\pi^{\prime}\right)$.

ii) For $l\left(C_{1}\right) \geqslant 2$ and $l\left(C_{2}\right)=1$, we suppose that $n-1, \bar{n}$ appear in $\pi$, i.e.,

$$
\pi=\cdots\left(\ldots, a, n-1, \pi_{n-1}, \ldots\right) \cdots(\bar{n}) \cdots
$$

This yields that $\pi^{\prime}=\cdots\left(\ldots, a, n-1, \bar{n}, \pi_{n-1}, \ldots\right) \cdots$, and therefore $w(\pi)=-w\left(\pi^{\prime}\right)$ from the fact that $a<n-1>\pi_{n-1}$ and $\pi_{n}=\bar{n}$ in $\pi$, yet $a<n-1>\bar{n}<\pi_{n-1}$ in $\pi^{\prime}$. Similar consideration could be made when $n$ is a singleton and $\overline{n-1}$ lies in a cycle of length at least two.

The catch comes when $n$ is a singleton and $n-1$ lies in a cycle of length at least two, i.e.,

$$
\pi=\cdots\left(\ldots, a, n-1, \pi_{n-1}, \ldots\right) \cdots(n) \cdots .
$$

We could solve this case by introducing $\pi^{\prime}=\cdots\left(\ldots, a, \overline{n-1}, \bar{n}, \pi_{n-1}, \ldots\right) \cdots$, and it is easy to get $w(\pi)=-w\left(\pi^{\prime}\right)$. Similar consideration could be made when $\bar{n}$ is a singleton and $\overline{n-1}$ lies in a cycle of length at least two, as well as the case $l\left(C_{1}\right)=1$ and $l\left(C_{2}\right) \geqslant 2$.

iii) For $l\left(C_{1}\right) \geqslant 2$ and $l\left(C_{2}\right) \geqslant 2$, suppose that $n-1$ and $\bar{n}$ appear in $\pi$ simultaneously, i.e.,

$$
\pi=\cdots\left(\ldots, a, n-1, \pi_{n-1}, \ldots\right) \cdots\left(\ldots, b, \bar{n}, \pi_{n}, \ldots\right) \cdots,
$$

which gives $\pi^{\prime}=\cdots\left(\ldots, a, n-1, \pi_{n}, \ldots, b, \bar{n}, \pi_{n-1}, \ldots\right) \cdots$. Further, we get $w(\pi)=$ $-w\left(\pi^{\prime}\right)$ from the only difference between the excedance set of $\pi$ and that of $\pi^{\prime}$, i.e., $n-1>\pi_{n-1}, \bar{n}<\pi_{n}$ in $\pi$ and $n-1>\pi_{n}, \bar{n}<\pi_{n-1}$ in $\pi^{\prime}$. Similar analysis could be made to the other three cases according to the signs of $n-1$ and $n$.

We end this subsection with two illustrative examples. For $\pi=(\overline{1} 4)(2 \overline{7} 6)(\overline{3} 58)$, we have $\pi_{7}=6, \pi_{8}=\overline{3}, \varphi(\pi)=(\overline{3}, 6) \circ \pi=(\overline{1} 4)(2 \overline{7} \overline{3} 586)$, and therefore $w(\pi)=-x^{4}$ and $w(\varphi(\pi))=x^{4}$; for $\pi=(1 \overline{3} 6)(2 \overline{5} 84)(\overline{7})$, we have $\varphi(\pi)=(\overline{7}, 4) \circ \pi=(1 \overline{3} 6)(2 \overline{5} 8 \overline{7} 4)$, and this gives $w(\pi)=-x^{3}$ and $w(\varphi(\pi))=x^{3}$.

\subsection{Combinatorial proof of the closed formula (3)}

For $1 \leqslant k \leqslant n$, let $\Omega_{n, k}$ denote the set of type $B$ permutations $\pi \in B_{n}$ whose cycle decompositions consist of a $k$-cycle such that $\left(1<\pi(1)<\pi^{2}(1)<\cdots<\pi^{k-1}(1)\right)$ and $n-k$ positive singletons; or a $k$-cycle $\left(\overline{1}>\pi(1)>\pi^{2}(1)>\cdots>\pi^{k-1}(1)\right)$ and $n-k$ negative singletons. 
It is easy to see that the weights of the first and second kind $k$-cycle are $-x^{k-1}$ and $-x$, respectively; the weights of the positive and negative singleton are -1 and $-x$, respectively. Therefore, we can get the weight of $\Omega_{n}:=\bigcup_{k=1}^{n} \Omega_{n, k}$ as

$$
\begin{aligned}
w\left(\Omega_{n}\right) & =\sum_{k=1}^{n}\left(\begin{array}{l}
n-1 \\
k-1
\end{array}\right)(-1)^{n-k+1} x^{k-1}+\sum_{k=1}^{n}\left(\begin{array}{c}
n-1 \\
k-1
\end{array}\right)(-1)^{n-k+1} x^{n-k+1} \\
& =-\sum_{k=0}^{n-1}\left(\begin{array}{c}
n-1 \\
k
\end{array}\right)(-1)^{n-1-k} x^{k}-x^{n} \sum_{k=0}^{n-1}\left(\begin{array}{c}
n-1 \\
k
\end{array}\right)(-1)^{n-1-k} x^{-k} \\
& =-(x-1)^{n-1}-x^{n}\left(x^{-1}-1\right)^{n-1} \\
& =(x-1)^{n-1}\left((-1)^{n} x-1\right) .
\end{aligned}
$$

To prove Eq. (3), it remains to construct a sign-reversing involution $\chi$ on $\bar{\Omega}_{n}:=B_{n} \backslash \Omega_{n}$.

Given $\pi \in \bar{\Omega}_{n}$, if all the elements of $\pi$ are positive, then we could use the involution provided in [12] for the permutations in $\mathfrak{S}_{n}$; and if all the elements are negative, we can use the known involution on $|\pi|$, and then change every element to its negative. Therefore, we only need to consider the case that positive and negative elements appear in $\pi$ simultaneously.

We first consider the case when 1 is positive in the cycle decomposition of $\pi$. Denote by $\bar{a}$ the smallest element in $\pi$, and set $m_{\pi}=\bar{a}$. We select another element $m_{\pi}^{\prime}$ as follows: Case 1: if there are no negative elements larger than $m_{\pi}$, then set $m_{\pi}^{\prime}=1$;

Case 2: if there are at least one negative elements in $\pi$ larger than $m_{\pi}$, then we denote by $\bar{b}$ the smallest element larger than $m_{\pi}$ in $\pi$, and

i) if $\pi_{b} \neq \bar{b}$ and $\pi_{b} \neq m_{\pi}$, then set $m_{\pi}^{\prime}=\bar{b}$;

ii) if $\pi_{b}=\bar{b}$, then find the smallest element larger than $\bar{b}$ in $\pi$, and check the condition i) until we find the right choice for $m_{\pi}^{\prime}$;

iii) if $\pi_{b}=m_{\pi}$, then we set $m_{\pi}=\bar{b}$, and check the conditions in Case 1 and Case 2, until we find the right choice for $m_{\pi}^{\prime}$.

With $m_{\pi}$ and $m_{\pi}^{\prime}$ in hand, we define

$$
\chi(\pi):=\left(m_{\pi}, m_{\pi}^{\prime}\right) \circ \pi,
$$

and will show that $\chi$ is a sign-reversing involution. Suppose $m_{\pi}=\bar{a}$ and $m_{\pi}^{\prime}=\bar{b}$, and thus $\bar{b}>\bar{a}$. Without loss of generality, assume that $m_{\pi}$ and $m_{\pi}^{\prime}$ are in different cycles, i.e.,

$$
\pi=\cdots\left(\bar{b}, \pi_{b}, \ldots, x\right) \cdots\left(\bar{a}, \pi_{a}, \ldots, y\right) \cdots .
$$

This gives $\chi(\pi)=\cdots\left(\bar{b}, \pi_{b}, \ldots, x, \bar{a}, \pi_{a}, \ldots, y\right) \cdots$. Observe the difference between the excedance sets of $\pi$ and $\chi(\pi)$, we see that $\bar{b}<x$ in $\pi$ (otherwise, $m_{\pi}^{\prime} \neq \bar{b}$ ), and thus $\bar{a}<x$ in $\chi(\pi)$. From the choice of $\bar{b}$, we have if $\bar{a}>y$ in $\pi$, then $\bar{b}>y$ in $\chi(\pi)$, and if $\bar{a}<y$ in $\pi$ then $\bar{b}<y$ in $\chi(\pi)$. Therefore, we get $w(\pi)=-w(\chi(\pi))$, and it is obvious 
that $m_{\chi(\pi)}=\bar{a}$ and $m_{\chi(\pi)}^{\prime}=\bar{b}$. We can also show that $\chi$ is a sign reversing and weight preserving involution when $\bar{a}$ is a singleton or $m_{\pi}^{\prime}=1$.

If $\overline{1}$ appears in $\pi$, then we introduce another sign-reversing involution $\chi^{\prime}$ by first changing the sign of every element in $\pi$ to obtain a new permutation $-\pi$; then applying the involution $\chi$ on $-\pi$; and at last $\chi^{\prime}(\pi)$ is obtained from $\chi(-\pi)$ by changing the sign of every element back. By combining the involutions $\chi$ and $\chi^{\prime}$, we see clearly that the weight of $\bar{\Omega}_{n}$ is zero, and this completes the proof.

We take an example to illustrate our proof. For $\pi=(\overline{1}, 2,5)(\overline{3}, 6,7, \overline{4})$, we have $-\pi=(1, \overline{2}, \overline{5})(3, \overline{6}, \overline{7}, 4), m_{-\pi}=\overline{6}, m_{-\pi}^{\prime}=\overline{5}$ and $\chi(-\pi)=(1, \overline{2}, \overline{6}, \overline{7}, 4,3, \overline{5})$. Thus, it holds that $\chi^{\prime}(\pi)=(\overline{1}, 2,6,7, \overline{4}, \overline{3}, 5), w(\pi)=x^{5}$ and $w\left(\chi^{\prime}(\pi)\right)=-x^{5}$.

Table 1 gives an illustration for $n=3$. For compactness, we only present those type $B$ permutations containing positive 1 and at least one negative elements.

\begin{tabular}{|c|c|c|c|c|}
\hline$\pi$ & $w(\pi)$ & $\left(m_{\pi}, m_{\pi}^{\prime}\right)$ & $w(\chi(\pi))$ & $\chi(\pi)$ \\
\hline \hline$(1, \overline{2}, 3)$ & $-x$ & $(1, \overline{2})$ & $x$ & $(1)(\overline{2}, 3)$ \\
\hline$(1,3, \overline{2})$ & $-x^{2}$ & $(1, \overline{2})$ & $x^{2}$ & $(1,3)(\overline{2})$ \\
\hline$(1,2, \overline{3})$ & $-x^{2}$ & $(1, \overline{3})$ & $x^{2}$ & $(1,2)(\overline{3})$ \\
\hline$(1, \overline{3}, 2)$ & $-x$ & $(1, \overline{3})$ & $x$ & $(1)(2, \overline{3})$ \\
\hline$(1, \overline{2}, \overline{3})$ & $-x$ & $(1, \overline{2})$ & $x$ & $(1)(\overline{2}, \overline{3})$ \\
\hline$(1, \overline{3}, \overline{2})$ & $-x^{2}$ & $(\overline{2}, \overline{3})$ & $x^{2}$ & $(1, \overline{2})(\overline{3})$ \\
\hline$(1, \overline{2})(3)$ & $x$ & $(1, \overline{2})$ & $-x$ & $(1)(\overline{2})(3)$ \\
\hline$(1, \overline{3})(2)$ & $x$ & $(1, \overline{3})$ & $-x$ & $(1)(2)(\overline{3})$ \\
\hline$(1, \overline{3})(\overline{2})$ & $x^{2}$ & $(1, \overline{3})$ & $-x^{2}$ & $(1)(\overline{2})(\overline{3})$ \\
\hline
\end{tabular}

Table 1: The involution $\chi$ for $n=3$.

\section{Signed excedances on type $B$ derangements}

In this section, we study the signed excedances on type $B$ derangements. By combinatorial arguments, we derive a recurrence and a closed formula for $P_{n}^{B}(x, 0,-1)$ as follows.

Theorem 4. For $n \geqslant 2$, we have

$$
\begin{aligned}
P_{n}^{B}(x, 0,-1) & =(2-x) P_{n-1}^{B}(x, 0,-1)-2 x^{n-1}, \\
P_{n}^{B}(x, 0,-1) & =\frac{x(2-x)^{n}-x^{n}}{x-1} \\
& =-\sum_{k=1}^{n} x\left(\begin{array}{l}
n \\
k
\end{array}\right)(1-x)^{k-1}-x-x^{2}-\cdots-x^{n-1},
\end{aligned}
$$

where $P_{1}^{B}(x, 0,-1)=-x$. 
Recall that $D_{n}^{B}$ represents the set of all type $B$ derangements on $[n]$. We denote by $d_{o}^{B}(n)$ and $d_{e}^{B}(n)$ the number of derangements in $D_{n}^{B}$ with odd and even number of cycles, respectively. By setting $x=1$ in Eq. (6), we have

Corollary 5. For $n \geqslant 1, d_{o}^{B}(n)-d_{e}^{B}(n)=2 n-1$.

A similar result for derangements in $\mathfrak{S}_{n}$ was given by Chapman [7], i.e., the number of even and odd derangements in $\mathfrak{S}_{n}$ differs by $n-1$. This result has been generalized by introducing the concept of "excedance" in the work of Mantaci and Rakotondrajao [13].

\subsection{Combinatorial proof of the recurrence (4)}

We first define four subsets of $D_{n}^{B}$ as follows:

- $D_{n}^{1}:=\left\{\pi \in D_{n}^{B} \mid\right.$ the relative positions of $n-1$ and $n$ are either $(\ldots, n, n-1, \ldots)$ or $(\ldots, \overline{n-1}, n, \ldots)$ except for the cycle $(n, n-1)\}$;

- $D_{n}^{2}:=\left\{\pi \in D_{n}^{B} \mid\right.$ the relative positions of $n-1$ and $n$ are either $(\ldots, \bar{n}, n-1, \ldots)$ or $(\ldots, \overline{n-1}, \bar{n}, \ldots)$ except for the cycle $(\bar{n}, n-1)\}$;

- $D_{n}^{3}:=\left\{\pi \in D_{n}^{B} \mid \pi_{n}=\bar{n}\right\}$;

- $D_{n}^{4}:=\{(1,2, \ldots, n-1, n),(1,2, \ldots, n-1, \bar{n})\}$.

We could construct a bijection between $D_{n}^{1}$ and $D_{n-1}^{B}$ by deleting the element $n$ from every permutation in $D_{n}^{1}$, and this bijection keeps the weight invariant. This means that the sets $D_{n}^{1}$ and $D_{n-1}^{B}$ have the same weight $P_{n-1}^{B}(x, 0,-1)$. Similarly, we can show that the weight of $D_{n}^{2}$ is also $P_{n-1}^{B}(x, 0,-1)$. It is obvious that the weights of $D_{n}^{3}$ and $D_{n}^{4}$ are equal to $-x P_{n-1}^{B}(x, 0,-1)$ and $-2 x^{n-1}$, respectively. Therefore, it remains to show that the weight of $\bar{D}_{n}^{B}:=D_{n}^{B} \backslash\left\{D_{n}^{1} \cup D_{n}^{2} \cup D_{n}^{3} \cup D_{n}^{4}\right\}$ equals to zero.

We will partition the set $\bar{D}_{n}^{B}$ into two classes according to the image of $n-1$ under the action of $\pi$, and construct the sign-reversing involution $\psi$ case by case.

Class A: For each permutation $\pi$ such that $\pi_{n-1} \neq n$ and $\pi_{n-1} \neq \bar{n}$, we define

$$
\psi(\pi):=\left(\pi_{n-1}, \pi_{n}\right) \circ \pi .
$$

Without loss of generality, we assume that $\pi_{n-1}$ and $\pi_{n}$ are in different cycles. According to the signs of $n-1$ and $n$ appear in $\pi$, we distinguish the following four cases to prove that $\psi$ is the desired involution.

A1: If $n-1$ and $n$ are the elements of $\pi$, i.e.,

$$
\pi=\cdots\left(\ldots, a, n-1, \pi_{n-1}, b, \ldots\right) \cdots\left(\ldots, x, n, \pi_{n}, y, \ldots\right) \cdots,
$$

then $\psi(\pi)=\cdots\left(\ldots, a, n-1, \pi_{n}, y, \ldots, x, n, \pi_{n-1}, b, \ldots,\right) \cdots$.

We have $\operatorname{exc}_{B}(\pi)=\operatorname{exc}_{B}(\psi(\pi))$ from the fact that $n-1>\pi_{n-1}, n>\pi_{n}$ in $\pi$, yet $n-1>\pi_{n}$ and $n>\pi_{n-1}$ in $\psi(\pi)$. 
A2: $\quad$ If $\overline{n-1}$ and $n$ are the elements of $\pi$ and $\pi_{n-1} \neq \overline{n-1}$, i.e.,

$$
\pi=\cdots\left(\ldots, a, \overline{n-1}, \pi_{n-1}, b, \ldots\right) \cdots\left(\ldots, x, n, \pi_{n}, y, \ldots\right) \cdots
$$

then $\psi(\pi)=\cdots\left(\ldots, a, \overline{n-1}, \pi_{n}, y, \ldots, x, n, \pi_{n-1}, b, \ldots,\right) \cdots$. From the fact that $\overline{n-1}<$ $\pi_{n-1}$ and $n>\pi_{n}$ in $\pi$, yet $\overline{n-1}<\pi_{n}$ and $n>\pi_{n-1}$ in $\psi(\pi)$, we have $\operatorname{exc}_{B}(\pi)=$ $\operatorname{exc}_{B}(\psi(\pi))$.

For $\pi_{n-1}=\overline{n-1}$, we have $\psi(\pi)=\cdots\left(\overline{n-1}, \pi_{n}, y, \ldots, x, n\right) \cdots$, and it is easy to see that $\operatorname{exc}_{B}(\pi)=\operatorname{exc}_{B}(\psi(\pi))$ since $\pi_{n-1}=\overline{n-1}$ and $n>\pi_{n}$ in $\pi$, yet $\overline{n-1}<\pi_{n}$ and $n>\overline{n-1}$ in $\psi(\pi)$.

A3: $\quad$ If $n-1$ and $\bar{n}$ are the elements of $\pi$, i.e.,

$$
\pi=\cdots\left(\ldots, a, n-1, \pi_{n-1}, b, \ldots\right) \cdots\left(\ldots, x, \bar{n}, \pi_{n}, y, \ldots\right) \cdots
$$

then $\psi(\pi)=\cdots\left(\ldots, a, n-1, \pi_{n}, y, \ldots, x, \bar{n}, \pi_{n-1}, b, \ldots,\right) \cdots$.

We have $\operatorname{exc}_{B}(\pi)=\operatorname{exc}_{B}(\psi(\pi))$ from the observation $n-1>\pi_{n-1}$ and $\bar{n}<\pi_{n}$ in $\pi$, while $n-1>\pi_{n}$ and $\bar{n}<\pi_{n-1}$ in $\psi(\pi)$.

A4: If $\overline{n-1}$ and $\bar{n}$ are the elements of $\pi$ and $\pi_{n-1} \neq \overline{n-1}$, i.e.,

$$
\pi=\cdots\left(\ldots, a, \overline{n-1}, \pi_{n-1}, b, \ldots\right) \cdots\left(\ldots, x, \bar{n}, \pi_{n}, y, \ldots\right) \cdots
$$

then $\psi(\pi)=\cdots\left(\ldots, a, \overline{n-1}, \pi_{n}, y, \ldots, x, \bar{n}, \pi_{n-1}, b, \ldots,\right) \cdots$.

We have $\overline{n-1}<\pi_{n-1}$ and $\bar{n}<\pi_{n}$ in $\pi$, yet $\overline{n-1}<\pi_{n}$ and $\bar{n}<\pi_{n-1}$ in $\psi(\pi)$, thus $\operatorname{exc}_{B}(\pi)=\operatorname{exc}_{B}(\psi(\pi))$.

For $\pi_{n-1}=\overline{n-1}$, then $\psi(\pi)=\cdots\left(\overline{n-1}, \pi_{n}, y, \ldots, x, \bar{n}\right) \cdots$. It holds that $\overline{n-1}<\pi_{n}$ from $\pi \notin D_{n}^{3}$ (that is $\pi_{n} \neq \bar{n}$ ). Thus, we have $\operatorname{exc}_{B}(\pi)=\operatorname{exc}_{B}(\psi(\pi))$ from the fact $\pi_{n-1}=\overline{n-1}$ and $\bar{n}<\pi_{n}$ in $\pi$, while $\overline{n-1}<\pi_{n}$ and $\bar{n}<\overline{n-1}$ in $\psi(\pi)$.

Class B: For each permutation $\pi$ such that $\pi_{n-1}=n$ or $\pi_{n-1}=\bar{n}$, the involution $\psi$ will be defined accordingly based on the signs of $n-2$ and $n$. We assume that $\pi_{n-2}$ and $\pi_{n}$ are in different cycles without loss of generality.

B1: If $n-2$ and $n$ are the elements of $\pi$, i.e.,

$$
\pi=\cdots\left(\ldots, x, n-1, n, \pi_{n}, \ldots\right) \cdots\left(\ldots, y, n-2, \pi_{n-2}, \ldots\right) \cdots
$$

and if $\pi_{n-2} \neq n-1$, then we define $\psi(\pi):=(n-2, n-1) \circ \pi$, i.e.,

$$
\psi(\pi)=\cdots\left(\ldots, x, n-2, \pi_{n-2}, \ldots, y, n-1, n, \pi_{n}, \ldots\right) \cdots .
$$

We have $\operatorname{exc}_{B}(\pi)=\operatorname{exc}_{B}(\psi(\pi))$ from the observation that $x<n-1$ and $y<n-2$ in $\pi$, yet $x<n-2$ and $y<n-1$ in $\psi(\pi)$. If $x=n$, we can also verify that $\operatorname{exc}_{B}(\pi)=\operatorname{exc}_{B}(\psi(\pi))$ by similar analysis.

For the case $\pi_{n-2}=n-1$, we define $\psi(\pi)=(j-1, j) \circ \pi$, where $j$ is the largest element such that $\pi_{j-1} \neq j$ and its existence is guaranteed from $\pi \notin D_{n}^{4}$. Here we omit the detailed proof since it is rather similar to the proof of the case when $\pi_{n-2} \neq n-1$. 
B2: If $\overline{n-2}$ and $n$ are the elements of $\pi$, i.e.,

$$
\pi=\cdots\left(\ldots, x, n-1, n, \pi_{n}, \ldots\right) \cdots\left(\ldots, y, \overline{n-2}, \pi_{n-2}, \ldots\right) \cdots,
$$

then we define $\psi(\pi):=\left(\pi_{n-2}, \pi_{n}\right) \circ \pi$, that is

$$
\psi(\pi)=\cdots\left(\ldots, x, n-1, n, \pi_{n-2}, \ldots, y, \overline{n-2}, \pi_{n}, \ldots\right) \cdots .
$$

For $\pi_{n} \neq \overline{n-2}$, we can easily obtain $\operatorname{exc}_{B}(\pi)=\operatorname{exc}_{B}(\psi(\pi))$ from the obvious fact $n>\pi_{n}, \overline{n-2}<\pi_{n-2}$ in $\pi$, while $n>\pi_{n-2}$ and $\overline{n-2}<\pi_{n}$ in $\psi(\pi)$.

For $\pi_{n}=\overline{n-2}$, we have $\pi=\cdots\left(\ldots, x, n-1, n, \overline{n-2}, \pi_{n-2}, \ldots\right) \cdots$, and it holds that $\psi(\pi)=\cdots\left(\ldots, x, n-1, n, \pi_{n-2}, \ldots\right) \cdots(\overline{n-2}) \cdots$. Thus, $\operatorname{exc}_{B}(\pi)=\operatorname{exc}_{B}(\psi(\pi))$ follows from $n>\overline{n-2}<\pi_{n-2}$ in $\pi$, while $n>\pi_{n-2}$ and $\pi_{n-2}=\overline{n-2}$ in $\psi(\pi)$.

B3: If $n-2$ and $\bar{n}$ are the elements of $\pi$, i.e.,

$$
\pi=\cdots\left(\ldots, x, n-1, \bar{n}, \pi_{n}, \ldots\right) \cdots\left(\ldots, y, n-2, \pi_{n-2}, \ldots\right) \cdots,
$$

and if $\pi_{n-2} \neq n-1$, then we define $\psi(\pi):=(n-2, n-1) \circ \pi$, i.e.,

$$
\psi(\pi)=\cdots\left(\ldots, x, n-2, \pi_{n-2}, \ldots, y, n-1, \bar{n}, \pi_{n}, \ldots\right) \cdots .
$$

We have $\operatorname{exc}_{B}(\pi)=\operatorname{exc}_{B}(\psi(\pi))$ from the fact that $x<n-2<n-1$ and $y<n-2<n-1$. If $x=\bar{n}$, we could also have $\operatorname{exc}_{B}(\pi)=\operatorname{exc}_{B}(\psi(\pi))$ from $y<n-2<n-1$ and $\bar{n}<n-2<n-1$.

For $\pi_{n-2}=n-1$, we could give a similar argument as that in case B1 by finding the largest element $j$ s.t. $\pi_{j-1} \neq j$ and then defining $\psi(\pi)=(j-1, j) \circ \pi$.

B4: If $\overline{n-2}$ and $\bar{n}$ are the elements of $\pi$, i.e.,

$$
\pi=\cdots\left(\ldots, x, n-1, \bar{n}, \pi_{n}, \ldots\right) \cdots\left(\ldots, y, \overline{n-2}, \pi_{n-2}, \ldots\right) \cdots,
$$

then we define $\psi(\pi):=\left(\pi_{n-2}, \pi_{n}\right) \circ \pi$, that is

$$
\psi(\pi)=\cdots\left(\ldots, x, n-1, \bar{n}, \pi_{n-2}, \ldots, y, \overline{n-2}, \pi_{n}, \ldots\right) \cdots .
$$

For $\pi_{n} \neq \overline{n-2}$, we have $\operatorname{exc}_{B}(\pi)=\operatorname{exc}_{B}(\psi(\pi))$ from the obvious fact $\bar{n}<\pi_{n}$ and $\overline{n-2}<\pi_{n-2}$ in $\pi$, while $\bar{n}<\pi_{n-2}$ and $\overline{n-2}<\pi_{n}$ in $\psi(\pi)$.

For $\pi_{n}=\overline{n-2}$, we have $\pi=\cdots\left(\ldots, x, n-1, \bar{n}, \overline{n-2}, \pi_{n-2}, \ldots\right) \cdots$, it holds that $\psi(\pi)=\cdots\left(\ldots, x, n-1, \bar{n}, \pi_{n-2}, \ldots\right) \cdots(\overline{n-2}) \cdots . \operatorname{Thus} \operatorname{exc}_{B}(\pi)=\operatorname{exc}_{B}(\psi(\pi))$ follows from $\bar{n}<\overline{n-2}<\pi_{n-2}$ and $\pi_{n-2}=\overline{n-2}$.

By combining the $\psi$ defined in class A and class B, we obtain the desired sign-reversing involution on set $\bar{D}_{n}^{B}$, and the weight of set $\bar{D}_{n}^{B}$ exactly equals to zero. This completes the proof.

For the special case $D_{3}^{B}$, we have

$$
\begin{aligned}
& D_{3}^{1}=\{(1,3,2),(\overline{1}, 3,2),(1, \overline{2}, 3),(\overline{1}, \overline{2}, 3),(\overline{1})(\overline{2}, 3)\}, \\
& D_{3}^{2}=\{(1, \overline{3}, 2),(\overline{1}, \overline{3}, 2),(1, \overline{2}, \overline{3}),(\overline{1}, \overline{2}, \overline{3}),(\overline{1})(\overline{2}, \overline{3})\}, \\
& D_{3}^{3}=\{(1,2)(\overline{3}),(1, \overline{2})(\overline{3}),(\overline{1}, 2)(\overline{3}),(\overline{1}, \overline{2})(\overline{3}),(\overline{1})(\overline{2})(\overline{3})\}, \\
& D_{3}^{4}=\{(1,2,3),(1,2, \overline{3})\},
\end{aligned}
$$

and Table 2 exhibits the involution $\psi$ on the set $\bar{D}_{3}^{B}$ in detail. 


\begin{tabular}{|c|c|c|c|c|}
\hline$\pi$ & $w(\pi)$ & transposition & $w(\psi(\pi))$ & $\psi(\pi)$ \\
\hline \hline$(\overline{1}, 2,3)$ & $-x^{2}$ & $\left(\pi_{1}, \pi_{3}\right)=(\overline{1}, 2)$ & $x^{2}$ & $(\overline{1})(2,3)$ \\
\hline$(\overline{1}, 2, \overline{3})$ & $-x^{2}$ & $\left(\pi_{1}, \pi_{3}\right)=(\overline{1}, 2)$ & $x^{2}$ & $(\overline{1})(2, \overline{3})$ \\
\hline$(1,3, \overline{2})$ & $-x^{2}$ & $\left(\pi_{2}, \pi_{3}\right)=(1, \overline{2})$ & $x^{2}$ & $(1,3)(\overline{2})$ \\
\hline$(\overline{1}, 3, \overline{2})$ & $-x^{2}$ & $\left(\pi_{2}, \pi_{3}\right)=(\overline{1}, \overline{2})$ & $x^{2}$ & $(\overline{1}, 3)(\overline{2})$ \\
\hline$(1, \overline{3}, \overline{2})$ & $-x^{2}$ & $\left(\pi_{2}, \pi_{3}\right)=(1, \overline{2})$ & $x^{2}$ & $(1, \overline{3})(\overline{2})$ \\
\hline$(\overline{1}, \overline{3}, \overline{2})$ & $-x^{2}$ & $\left(\pi_{2}, \pi_{3}\right)=(\overline{1}, \overline{2})$ & $x^{2}$ & $(\overline{1}, \overline{3})(\overline{2})$ \\
\hline
\end{tabular}

Table 2: The involution $\psi$ on the set $\bar{D}_{3}^{B}$.

\subsection{Combinatorial proof of the closed formula (6)}

We begin with the following theorem, which is a refinement of the formula (6).

Theorem 6. For $0 \leqslant k \leqslant n$, let $D_{n, k}^{B}=\left\{\pi \in D_{n}^{B} \mid \pi\right.$ has exactly $k$ negative elements $\}$. Then

$$
\sum_{\pi \in D_{n, 0}^{B}}(-1)^{\mathrm{cyc}(\pi)} x^{\operatorname{exc}_{B}(\pi)}=-x-x^{2}-\cdots-x^{n-1}
$$

and for $k \geqslant 1$,

$$
\sum_{\pi \in D_{n, k}^{B}}(-1)^{\operatorname{cyc}(\pi)} x^{\operatorname{exc}_{B}(\pi)}=-x\left(\begin{array}{l}
n \\
k
\end{array}\right)(1-x)^{k-1} .
$$

Proof. We will focus on the general cases for $D_{n, k}^{B}$ with $k \geqslant 1$ because Ksavrelof and Zeng [12] have presented detailed proofs for $D_{n, 0}^{B}$.

The set of type $B$ permutations with exactly $k$ negative elements is isomorphic to the set of type $B$ permutations with negative elements $\overline{1}, \overline{2}, \ldots, \bar{k}$, we only need to consider the subset $\mathcal{D}_{n, k}^{B}$ of $D_{n, k}^{B}$ whose elements are derangements with bars only occur on elements $1,2, \ldots, k$. Hence, to prove the weight of set $D_{n, k}^{B}$ is $-x\left(\begin{array}{l}n \\ k\end{array}\right)(1-x)^{k-1}$, it remains to prove that the weight of set $\mathcal{D}_{n, k}^{B}$ is $-x(1-x)^{k-1}$.

For $1<k<n$, we denote by $\mathcal{O}_{n, k}^{B}$ the subset of $\mathcal{D}_{n, k}^{B}$ such that:

a) $\overline{1}$ and $n$ are in the same cycle, and $n$ lies to the right of $\overline{1}$;

b) for $2 \leqslant i \leqslant k$, either $\bar{i}$ is a singleton, or $\bar{i}$ lies in the cycle containing $\overline{1}$ and stays immediately right after the largest (in absolute value) element smaller than $i$ of this cycle;

c) for $k<i<n, i$ stays in the cycle containing $\overline{1}$ and follows immediately right after the element $i+1$. 
For example, if $n=5$ and $k=2$, then $\mathcal{O}_{5,2}^{B}$ only has two permutations: $(\overline{1}, \overline{2}, 5,4,3)$ and $(\overline{1}, 5,4,3)(\overline{2})$. For $k=1$, it is sufficient for the subset $\mathcal{O}_{n, 1}^{B}$ of $\mathcal{D}_{n, 1}^{B}$ to satisfy the rules a) and c); and for $k=n$, it is sufficient for the subset $\mathcal{O}_{n, n}^{B}$ of $\mathcal{D}_{n, n}^{B}$ to satisfy the rule b).

Note that the weight of the cycle containing $\overline{1}$ is always $-x$ by the above construction, and for the element $\bar{i}(2 \leqslant i \leqslant k)$, it contributes weight $1-x$ since $\bar{i}$ maybe a singleton or in the cycle containing $\overline{1}$, so the weight of $\mathcal{O}_{n, k}^{B}(1 \leqslant k \leqslant n)$ is $-x(1-x)^{k-1}$. To prove that the weight of set $\mathcal{D}_{n, k}^{B}$ is $-x(1-x)^{k-1}$, we will construct a sign-reversing and weight preserving involution on $\overline{\mathcal{D}}_{n, k}^{B}:=\mathcal{D}_{n, k}^{B} \backslash \mathcal{O}_{n, k}^{B}$.

For $1 \leqslant k<n$, given a permutation $\pi \in \overline{\mathcal{D}}_{n, k}^{B}$, we define an involution $\phi$ on $\overline{\mathcal{D}}_{n, k}^{B}$ as follows:

Step1 If $\pi^{-1}(1) \neq k+1$, then define $\phi(\pi)=(\overline{1}, k+1) \circ \pi$; otherwise, go to Step2;

Step2 If there exists a smallest element $i(2 \leqslant i \leqslant k)$ such that $\pi_{i} \neq \bar{i}$ and $\pi^{-1}(i) \neq \overline{i-1}$, then we define $\phi(\pi)=\left(\pi_{j}, \pi_{i}\right) \circ \pi$, where $j$ is the largest integer smaller than $i$ satisfying $\pi_{j} \neq \bar{j}$; otherwise skip to Step3;

Step3 Define $\phi(\pi)=(i, i+1) \circ \pi$ by finding the smallest element $i(k<i<n)$ such that $\pi^{-1}(i) \neq i+1$.

Now we show that $\phi$ is a desired involution on $\overline{\mathcal{D}}_{n, k}^{B}$ according to the above three cases. 1) If $\pi^{-1}(1) \neq k+1$, i.e., $\pi=(\ldots, a, \overline{1}, b, \ldots) \cdots(\ldots, x, k+1, y, \ldots) \cdots$, then we have

$$
\phi(\pi)=(\overline{1}, b, \ldots, a, k+1, y, \ldots, x) \cdots
$$

and $\phi(\pi)^{-1}(1) \neq k+1$. It always holds that $\operatorname{exc}_{B}(\pi)=\operatorname{exc}_{B}(\phi(\pi))$ by considering the values of $a$ and $x$ :

i) if $a<\overline{1}$, then $a<k+1$;

ii) if $a>\overline{1}$, then $a>k+1$ from $a>0, a \neq k+1$ and $k+1$ being the smallest positive integer in $\pi$;

iii) if $x>k+1$, then $x>\overline{1}$;

iv) if $x<k+1$, then $x<\overline{1}$ from $x<0$ and $\overline{1}$ being the largest negative integer in $\pi$.

2) If $\pi^{-1}(1)=k+1$ and there exists a smallest $i(2 \leqslant i \leqslant k)$ s.t. $\pi_{i} \neq \bar{i}$ and $\pi^{-1}(i) \neq \overline{i-1}$, then $\pi=\left(\overline{1}, \ldots, \bar{j}, \pi_{j}, \ldots, k+1\right) \cdots(\bar{r}) \cdots\left(a, \ldots, \bar{i}, \pi_{i}, \ldots, b\right) \cdots$, where $j$ is the largest integer smaller than $i$ satisfying $\pi_{j} \neq \bar{j}$. Therefore,

$$
\phi(\pi)=\left(\overline{1}, \ldots, \bar{j}, \pi_{i}, \ldots, b, a, \ldots, \bar{i}, \pi_{j}, \ldots, k+1\right) \cdots(\bar{r}) \cdots .
$$

It is easy to see that $\phi(\pi)^{-1}(1)=k+1$, and $\bar{i}<\bar{j}$. We further have $\phi(\pi)(i)=\pi_{j} \neq \bar{i}$ from the choice of $i$ and $\phi(\pi)^{-1}(i) \neq \overline{i-1}$. By considering the values of $\pi_{i}$ and $\pi_{j}$, we see that i) if $\pi_{i}<0$ and $\pi_{j}<0$, then $\bar{j}>\bar{i}>\pi_{i}$ and $\bar{j}>\bar{i}>\pi_{j}$;

ii) if $\pi_{i}<0$ and $\pi_{j}>0$, then $\bar{j}>\bar{i}>\pi_{i}$ and $\bar{i}<\bar{j}<\pi_{j}$;

iii) if $\pi_{i}>0$ and $\pi_{j}<0$, then $\bar{i}<\bar{j}<\pi_{i}$ and $\bar{j}>\bar{i}>\pi_{j}$;

iv) if $\pi_{i}>0$ and $\pi_{j}>0$, then $\bar{i}<\bar{j}<\pi_{i}$ and $\bar{i}<\bar{j}<\pi_{j}$.

Thus it yields that $\operatorname{exc}_{B}(\pi)=\operatorname{exc}_{B}(\phi(\pi))$. 
3) if $\pi$ does not satisfy the conditions in Step1 and Step2, then there exists a smallest integer $i(k<i<n)$ such that $\pi^{-1}(i) \neq i+1$ from the definition of $\overline{\mathcal{D}}_{n, k}^{B}$. We assume

$$
\pi=\left(\overline{1}, \ldots, \bar{j}, \ldots, a, i+1, b, \ldots, \pi^{-1}(i), i, i-1, \ldots, k+1\right) \cdots(\bar{r}) \cdots,
$$

then $\phi(\pi)=(\overline{1}, \ldots, \bar{j}, \ldots, a, i, i-1, \ldots, k+1) \cdots\left(i+1, b, \ldots, \pi^{-1}(i)\right) \cdots(\bar{r}) \cdots$. It is easy to prove that $\phi(\pi)$ also belongs to the third case and $i$ is the smallest integer such that $\phi(\pi)^{-1}(i) \neq i+1$. On the other hand, we have $\operatorname{exc}_{B}(\pi)=\operatorname{exc}_{B}(\phi(\pi))$ by considering the values of $\pi^{-1}(i)$ and $a$ :

i) if $\pi^{-1}(i)>i$, then $\pi^{-1}(i)>i+1$ since $\pi^{-1}(i) \neq i+1$;

ii) if $\pi^{-1}(i)<i$, then $\pi^{-1}(i)<i+1$;

iii) if $a>0$, then $a>i+1>i$;

iv) if $a<0$, then $a<i<i+1$.

Hence $\phi$ is proven to be the desired involution on the set $\overline{\mathcal{D}}_{n, k}^{B}$ with $1 \leqslant k<n$.

While for the set $\overline{\mathcal{D}}_{n, n}^{B}$, the involution $\phi$ can be defined as $\phi(\pi)=\left(\pi_{j}, \pi_{i}\right) \circ \pi$, where $i$ is the smallest element such that $\pi_{i} \neq \bar{i}$ and $\pi^{-1}(i) \neq \overline{i-1}$, and $j$ is the largest integer smaller than $i$ satisfying $\pi_{j} \neq \bar{j}$. If no such $j$ exits, then let $j=1$.

If $j>1$, by the analysis in the case 2) for $k<n$, we see that $\phi$ is also the desired involution on the set $\overline{\mathcal{D}}_{n, n}^{B}$.

If $j=1$, suppose $\pi=(\overline{1}) \cdots(\bar{r}) \cdots\left(a, \ldots, \bar{i}, \pi_{i}, \ldots, b\right) \cdots$, then

$$
\phi(\pi)=\left(\overline{1}, \pi_{i}, \ldots, b, a, \ldots, \bar{i}\right) \cdots(\bar{r}) \cdots .
$$

By the choice of $i$, we see that $\bar{i}>\pi_{i}, \pi_{1}=\overline{1}$ in $\pi$, yet $\overline{1}>\pi_{i}, \bar{i}<\overline{1}$ in $\phi(\pi)$, thus $\operatorname{exc}_{B}(\pi)=\operatorname{exc}_{B}(\phi(\pi))$.

In summary, $\phi$ is a sign-reversing and weight preserving involution on the set $\overline{\mathcal{D}}_{n, k}^{B}$ for all $1 \leqslant k \leqslant n$, and this theorem follows.

We end this section with an example of $\phi$ on the set $\overline{\mathcal{D}}_{4, k}^{B}(1 \leqslant k \leqslant 4)$, and we also list those sets $\mathcal{O}_{4, k}^{B}$ for completeness.

i) $\mathcal{O}_{4,1}^{B}=\{(\overline{1}, 4,3,2)\}$, and the involution $\phi$ on subset $\overline{\mathcal{D}}_{4,1}^{B}$ is:

\begin{tabular}{|c|c|c|c|c|}
\hline$\pi$ & $w(\pi)$ & transposition & $w(\phi(\pi))$ & $\phi(\pi)$ \\
\hline \hline$(\overline{1}, 2,3,4)$ & $-x^{3}$ & $(\overline{1}, k+1)=(\overline{1}, 2)$ & $x^{3}$ & $(\overline{1})(2,3,4)$ \\
\hline$(\overline{1}, 2,4,3)$ & $-x^{2}$ & $(\overline{1}, k+1)=(\overline{1}, 2)$ & $x^{2}$ & $(\overline{1})(2,4,3)$ \\
\hline$(\overline{1}, 3,2,4)$ & $-x^{2}$ & $(\overline{1}, k+1)=(\overline{1}, 2)$ & $x^{2}$ & $(\overline{1}, 3)(2,4)$ \\
\hline$(\overline{1}, 4,2,3)$ & $-x^{2}$ & $(\overline{1}, k+1)=(\overline{1}, 2)$ & $x^{2}$ & $(\overline{1}, 4)(2,3)$ \\
\hline$(\overline{1}, 3,4,2)$ & $-x^{2}$ & $(i, i+1)=(2,3)$ & $x^{2}$ & $(\overline{1}, 2)(3,4)$ \\
\hline
\end{tabular}

ii) $\mathcal{O}_{4,2}^{B}=\{(\overline{1}, \overline{2}, 4,3),(\overline{1}, 4,3)(\overline{2})\}$, and the involution $\phi$ on subset $\overline{\mathcal{D}}_{4,2}^{B}$ is: 


\begin{tabular}{|c|c|c|c|c|}
\hline$\pi$ & $w(\pi)$ & transposition & $w(\phi(\pi))$ & $\phi(\pi)$ \\
\hline \hline$(\overline{1})(\overline{2})(3,4)$ & $-x^{3}$ & $(\overline{1}, k+1)=(\overline{1}, 3)$ & $x^{3}$ & $(\overline{1}, 3,4)(\overline{2})$ \\
\hline$(\overline{1}, 3,4, \overline{2})$ & $-x^{3}$ & $(\overline{1}, k+1)=(\overline{1}, 3)$ & $x^{3}$ & $(\overline{1})(\overline{2}, 3,4)$ \\
\hline$(\overline{1}, 3, \overline{2}, 4)$ & $-x^{2}$ & $(\overline{1}, k+1)=(\overline{1}, 3)$ & $x^{2}$ & $(\overline{1})(\overline{2}, 4,3)$ \\
\hline$(\overline{1}, \overline{2}, 3,4)$ & $-x^{2}$ & $(\overline{1}, k+1)=(\overline{1}, 3)$ & $x^{2}$ & $(\overline{1}, \overline{2})(3,4)$ \\
\hline$(\overline{1}, 4,3, \overline{2})$ & $-x^{2}$ & $(\overline{1}, k+1)=(\overline{1}, 3)$ & $x^{2}$ & $(\overline{1}, 4)(\overline{2}, 3)$ \\
\hline$(\overline{1}, 4, \overline{2}, 3)$ & $-x^{2}$ & $\left(\pi_{1}, \pi_{2}\right)=(3,4)$ & $x^{2}$ & $(\overline{1}, 3)(\overline{2}, 4)$ \\
\hline
\end{tabular}

iii) $\mathcal{O}_{4,3}^{B}=\{(\overline{1}, \overline{2}, \overline{3}, 4),(\overline{1}, \overline{2}, 4)(\overline{3}),(\overline{1}, \overline{3}, 4)(\overline{2}),(\overline{1}, 4)(\overline{2})(\overline{3})\}$, the involution $\phi$ on $\overline{\mathcal{D}}_{4,3}^{B}$ is:

\begin{tabular}{|c|c|c|c|c|}
\hline$\pi$ & $w(\pi)$ & transposition & $w(\phi(\pi))$ & $\phi(\pi)$ \\
\hline \hline$(\overline{1}, 4, \overline{3}, \overline{2})$ & $-x^{3}$ & $(\overline{1}, k+1)=(\overline{1}, 4)$ & $x^{3}$ & $(\overline{1})(\overline{2}, 4, \overline{3})$ \\
\hline$(\overline{1})(\overline{2}, 4)(\overline{3})$ & $-x^{3}$ & $(\overline{1}, k+1)=(\overline{1}, 4)$ & $x^{3}$ & $(\overline{1}, 4, \overline{2})(\overline{3})$ \\
\hline$(\overline{1})(\overline{2})(\overline{3}, 4)$ & $-x^{3}$ & $(\overline{1}, k+1)=(\overline{1}, 4)$ & $x^{3}$ & $(\overline{1}, 4, \overline{3})(\overline{2})$ \\
\hline$(\overline{1}, \overline{2}, 4, \overline{3})$ & $-x^{2}$ & $(\overline{1}, k+1)=(\overline{1}, 4)$ & $x^{2}$ & $(\overline{1}, \overline{2})(\overline{3}, 4)$ \\
\hline$(\overline{1}, \overline{3}, 4, \overline{2})$ & $-x^{2}$ & $(\overline{1}, k+1)=(\overline{1}, 4)$ & $x^{2}$ & $(\overline{1}, \overline{3})(\overline{2}, 4)$ \\
\hline$(\overline{1}, 4, \overline{2}, \overline{3})$ & $-x^{2}$ & $(\overline{1}, k+1)=(\overline{1}, 4)$ & $x^{2}$ & $(\overline{1})(\overline{2}, \overline{3}, 4)$ \\
\hline$(\overline{1}, \overline{3}, \overline{2}, 4)$ & $-x^{2}$ & $\left(\pi_{1}, \pi_{2}\right)=(\overline{3}, 4)$ & $x^{2}$ & $(\overline{1}, 4)(\overline{2}, 3)$ \\
\hline
\end{tabular}

iv) $\mathcal{O}_{4,4}^{B}=\{(\overline{1}, \overline{2}, \overline{3}, \overline{4}),(\overline{1}, \overline{2}, \overline{3})(\overline{4}),(\overline{1}, \overline{2}, \overline{4})(\overline{3}),(\overline{1}, \overline{3}, \overline{4})(\overline{2}),(\overline{1}, \overline{2})(\overline{3})(\overline{4}),(\overline{1}, \overline{3})(\overline{2})(\overline{4})$, $(\overline{1}, \overline{4})(\overline{2})(\overline{3}),(\overline{1})(\overline{2})(\overline{3})(\overline{4})\}$, and the involution $\phi$ on subset $\overline{\mathcal{D}}_{4,4}^{B}$ is:

\begin{tabular}{|c|c|c|c|c|}
\hline$\pi$ & $w(\pi)$ & transposition & $w(\phi(\pi))$ & $\phi(\pi)$ \\
\hline \hline$(\overline{1})(\overline{2})(\overline{3}, \overline{4})$ & $-x^{3}$ & $\left(\pi_{1}, \pi_{3}\right)=(\overline{1}, \overline{4})$ & $x^{3}$ & $(\overline{1}, \overline{4}, \overline{3})(\overline{2})$ \\
\hline$(\overline{1})(\overline{2}, \overline{3})(\overline{4})$ & $-x^{3}$ & $\left(\pi_{1}, \pi_{2}\right)=(\overline{1}, \overline{3})$ & $x^{3}$ & $(\overline{1}, \overline{3}, \overline{2})(\overline{4})$ \\
\hline$(\overline{1})(\overline{2}, \overline{4})(\overline{3})$ & $-x^{3}$ & $\left(\pi_{1}, \pi_{2}\right)=(\overline{1}, \overline{4})$ & $x^{3}$ & $(\overline{1}, \overline{4}, \overline{2})(\overline{3})$ \\
\hline$(\overline{1}, \overline{4}, \overline{3}, \overline{2})$ & $-x^{3}$ & $\left(\pi_{1}, \pi_{2}\right)=(\overline{1}, \overline{4})$ & $x^{3}$ & $(\overline{1})(\overline{2}, \overline{4}, \overline{3})$ \\
\hline$(\overline{1}, \overline{2}, \overline{4}, \overline{3})$ & $-x^{2}$ & $\left(\pi_{2}, \pi_{3}\right)=(\overline{1}, \overline{4})$ & $x^{2}$ & $(\overline{1}, \overline{2})(\overline{3}, \overline{4})$ \\
\hline$(\overline{1}, \overline{3}, \overline{4}, \overline{2})$ & $-x^{2}$ & $\left(\pi_{1}, \pi_{2}\right)=(\overline{1}, \overline{3})$ & $x^{2}$ & $(\overline{1})(\overline{2}, \overline{3}, \overline{4})$ \\
\hline$(\overline{1}, \overline{3}, \overline{2}, \overline{4})$ & $-x^{2}$ & $\left(\pi_{1}, \pi_{2}\right)=(\overline{3}, \overline{4})$ & $x^{2}$ & $(\overline{1}, \overline{4})(\overline{2}, \overline{3})$ \\
\hline$(\overline{1}, \overline{4}, \overline{2}, \overline{3})$ & $-x^{2}$ & $\left(\pi_{1}, \pi_{2}\right)=(\overline{3}, \overline{4})$ & $x^{2}$ & $(\overline{1}, \overline{3})(\overline{2}, \overline{4})$ \\
\hline
\end{tabular}

\section{The recurrence and generating function formula for the polynomial $P_{n}^{B}(x, y, z)$}

In this section, we derive the recurrence relation of $P_{n}^{B}(x, y, z)$ based on combinatorial arguments, and further present new relationships between the number $P_{n}^{B}(-1,1,1)$ and Euler number, as well as the relationships between $P_{n}^{B}(-1,0,1)$ and Springer number. 


\subsection{Recurrence formula for the polynomial $P_{n}^{B}(x, y, z)$}

Theorem 7. For $n \geqslant 1$, the polynomial $P_{n}^{B}(x, y, z)$ satisfies the recursion

$$
\begin{aligned}
P_{n}^{B}(x, y, z)= & {[(2 n-2+z) x+y z] P_{n-1}^{B}(x, y, z) } \\
& +2\left[x(1-x) \frac{\partial}{\partial x}+x(1-y) \frac{\partial}{\partial y}\right] P_{n-1}^{B}(x, y, z) .
\end{aligned}
$$

Proof. This theorem holds obviously for $n=1$ from $P_{0}^{B}(x, y, z)=1$ and $P_{1}^{B}(x, y, z)=$ $x z+y z$. For $n \geqslant 2$ and $\pi=\pi_{1} \pi_{2} \cdots \pi_{n-1} \in B_{n-1}$, let $\pi^{(i)}$ (resp., $\pi^{(\bar{i})}$ ) be the type $B$ permutation obtained by inserting $n$ (resp., $\bar{n}$ ) just before $\pi_{i}$ in the cycle decomposition of $\pi$ for $1 \leqslant i \leqslant n-1$, and $\pi^{(n)}$ (resp., $\pi^{(\bar{n})}$ ) is the type $B$ permutation derived by adding a cycle $(n)$ (resp., $(\bar{n})$ ) into $\pi$. It is easy to get $\operatorname{exc}_{B}\left(\pi^{(n)}\right)=\operatorname{exc}_{B}(\pi)$ and $\operatorname{fix}\left(\pi^{(n)}\right)=$ $\operatorname{fix}(\pi)+1$. For $i \in[n-1]$, we have

$$
\operatorname{exc}_{B}\left(\pi^{(i)}\right)=\left\{\begin{array}{ll}
\operatorname{exc}_{B}(\pi), & \text { if } \pi_{i}>i, \\
\operatorname{exc}_{B}(\pi)+1, & \text { if } \pi_{i} \leqslant i,
\end{array} \quad \operatorname{fix}\left(\pi^{(i)}\right)= \begin{cases}\operatorname{fix}(\pi), & \text { if } \pi_{i} \neq i \\
\operatorname{fix}(\pi)-1, & \text { if } \pi_{i}=i\end{cases}\right.
$$

For $i \in[n]$, we have

$$
\operatorname{cyc}\left(\pi^{(i)}\right)= \begin{cases}\operatorname{cyc}(\pi), & \text { if } i<n, \\ \operatorname{cyc}(\pi)+1, & \text { if } i=n .\end{cases}
$$

It is noteworthy that similar results hold for $\pi^{(\bar{i})}$, and we have

$$
\begin{aligned}
& \sum_{i=1}^{n} \sum_{\pi \in B_{n-1}}\left(x^{\operatorname{exc}_{B}\left(\pi^{(i)}\right)} y^{\mathrm{fix}\left(\pi^{(i)}\right)} z^{\mathrm{cyc}\left(\pi^{(i)}\right)}+x^{\operatorname{exc}_{B}\left(\pi^{(\bar{i})}\right)} y^{\mathrm{fix}\left(\pi^{(\bar{i})}\right)} z^{\operatorname{cyc}\left(\pi^{(\bar{i})}\right)}\right) \\
= & \sum_{i=1}^{n-1} \sum_{\pi \in B_{n-1}}\left(x^{\operatorname{exc}_{B}\left(\pi^{(i)}\right)} y^{\operatorname{fix}\left(\pi^{(i)}\right)} z^{\operatorname{cyc}\left(\pi^{(i)}\right)}+x^{\operatorname{exc}_{B}\left(\pi^{(\bar{i})}\right)} y^{\operatorname{fix}\left(\pi^{(\bar{i})}\right)} z^{\operatorname{cyc}\left(\pi^{(\bar{i})}\right)}\right) \\
& +\sum_{\pi \in B_{n-1}}\left(x^{\operatorname{exc}_{B}(\pi)} y^{\operatorname{fix}(\pi)+1} z^{\operatorname{cyc}(\pi)+1}+x^{\operatorname{exc}_{B}(\pi)+1} y^{\mathrm{fix}(\pi)} z^{\operatorname{cyc}(\pi)+1}\right) \\
= & 2 \sum_{\pi \in B_{n-1}}\left\{\operatorname{exc}_{B}(\pi) x^{\operatorname{exc}_{B}(\pi)} y^{\operatorname{fix}(\pi)} z^{\operatorname{cyc}(\pi)}+\operatorname{fix}(\pi) x^{\operatorname{exc}_{B}(\pi)+1} y^{\mathrm{fix}(\pi)-1} z^{\operatorname{cyc}(\pi)}\right. \\
& \left.+\left(n-1-\operatorname{exc}_{B}(\pi)-\operatorname{fix}(\pi)\right) x^{\operatorname{exc} B(\pi)+1} y^{\operatorname{fix}(\pi)} z^{\operatorname{cyc}(\pi)}\right\}+(x+y) z P_{n-1}^{B}(x, y, z) \\
= & (x+y) z P_{n-1}^{B}(x, y, z)+2 \sum_{\pi \in B_{n-1}}\left\{(1-x) \operatorname{exc}_{B}(\pi) x^{\operatorname{exc}_{B}(\pi)} y^{\mathrm{fix}(\pi)} z^{\operatorname{cyc}(\pi)}\right. \\
& \left.+x(1-y) \operatorname{fix}(\pi) x^{\operatorname{exc}_{B}(\pi)} y^{\operatorname{fix}(\pi)-1} z^{\operatorname{cyc}(\pi)}+(n-1) x^{\operatorname{exc}_{B}(\pi)+1} y^{\mathrm{fix}(\pi)} z^{\operatorname{cyc}(\pi)}\right\} .
\end{aligned}
$$

Now we can derive the recurrence for $P_{n}^{B}(x, y, z)=\sum_{\pi \in B_{n}} x^{\operatorname{exc}_{B}(\pi)} y^{\mathrm{fix}(\pi)} z^{\mathrm{cyc}(\pi)}$ as

$$
\begin{aligned}
P_{n}^{B}(x, y, z)= & (x+y) z P_{n-1}^{B}(x, y, z)+2\left[\left(x-x^{2}\right) \frac{\partial}{\partial x} P_{n-1}^{B}(x, y, z)\right. \\
& \left.+(x-x y) \frac{\partial}{\partial y} P_{n-1}^{B}(x, y, z)+(n-1) x P_{n-1}^{B}(x, y, z)\right] .
\end{aligned}
$$

Thus the theorem follows by collecting similar terms. 
If we weight each type $B$ permutation $\pi \in B_{n}$ by $w(\pi)=x^{\operatorname{exc}_{B}(\pi)} y^{\operatorname{fix}(\pi)}(-1)^{\operatorname{cyc}(\pi)}$, then we have the counting polynomial $(-y)^{k} P_{n-k}^{B}(x, 0,-1)$ for the total weight of permutations in $B_{n}$ with $k(0 \leqslant k \leqslant n)$ chosen fixed points. From Eq. (5), we further get

$$
P_{n}^{B}(x, y,-1)=\sum_{k=0}^{n}\left(\begin{array}{l}
n \\
k
\end{array}\right)(-y)^{k} P_{n-k}^{B}(x, 0,-1)=\frac{x(2-x-y)^{n}-(x-y)^{n}}{x-1} .
$$

We could also obtain the following proposition from the combinatorial interpretation of functional composition of exponential generating function[15, Chap. 5].

Proposition 8. For $n \geqslant 0$, we have

$$
\sum_{n \geqslant 0} P_{n}^{B}(x, y, z) \frac{t^{n}}{n !}=\left(\frac{(1-x) e^{(x+y) t}}{e^{2 x t}-x e^{2 t}}\right)^{z} .
$$

Proof. Since every type $B$ permutation is generated by cycles, we have

$$
\begin{aligned}
& \sum_{n \geqslant 0} P_{n}^{B}(x, y, z) \frac{t^{n}}{n !}=\exp \left(\sum_{n \geqslant 1} \sum_{\pi \in \mathcal{C}_{n}} x^{\operatorname{exc}_{B}(\pi)} y^{\mathrm{fix}(\pi)} z^{\operatorname{cyc}(\pi)} \frac{t^{n}}{n !}\right) \\
= & \exp \left(z \sum_{n \geqslant 1} \sum_{\pi \in \mathcal{C}_{n}} x^{\operatorname{exc}_{B}(\pi)} y^{\mathrm{fix}(\pi)} \frac{t^{n}}{n !}\right)=\left[\exp \left(-\sum_{n \geqslant 1} \sum_{\pi \in \mathcal{C}_{n}} x^{\operatorname{exc}_{B}(\pi)} y^{\mathrm{fix}(\pi)} \frac{t^{n}}{n !}\right)\right]^{-z} \\
= & {\left[\exp \left(\sum_{n \geqslant 1} \sum_{\pi \in \mathcal{C}_{n}} x^{\operatorname{exc}_{B}(\pi)} y^{\mathrm{fix}(\pi)}(-1)^{\operatorname{cyc}(\pi)} \frac{t^{n}}{n !}\right)\right]^{-z}=\left[1+\sum_{n \geqslant 1} P_{n}^{B}(x, y,-1) \frac{t^{n}}{n !}\right]^{-z}, }
\end{aligned}
$$

where $\mathcal{C}_{n}$ denotes the set of permutations $\pi \in B_{n}$ with $\operatorname{cyc}(\pi)=1$. We complete the proof by substituting the formula (9) into the last expression and simplifying it with the formula $e^{x}=\sum_{n \geqslant 0} x^{n} / n$ !.

The generating function for $P_{n}^{B}(x, y, z)$ is derived based on the known formula for $P_{n}^{B}(x, y,-1)$. From the above analysis that every type $B$ permutation is generated by cycles, we can also obtain Eq. (10) by the formula

$$
\sum_{n \geqslant 0} P_{n}^{B}(x, y, z) \frac{t^{n}}{n !}=\left[\sum_{n \geqslant 0} P_{n}^{B}(x, y, 1) \frac{t^{n}}{n !}\right]^{z} .
$$

Exploring the generating function of the derangement polynomials derived by Chow [11] and Chen et al. [8], we have

$$
\sum_{n \geqslant 0} P_{n}^{B}(x, 0,1) \frac{t^{n}}{n !}=\frac{(1-x) e^{x t}}{e^{2 x t}-x e^{2 t}} .
$$

Since a type $B$ permutation can be seen as disjoint union of fixed points and derangements, it is easy to derive

$$
\sum_{n \geqslant 0} P_{n}^{B}(x, y, 1) \frac{t^{n}}{n !}=\left(\sum_{n \geqslant 0} y^{n} \frac{t^{n}}{n !}\right)\left(\sum_{n \geqslant 0} P_{n}^{B}(x, 0,1) \frac{t^{n}}{n !}\right)=\frac{(1-x) e^{(x+y) t}}{e^{2 x t}-x e^{2 t}}
$$

which implies Eq. (10). 


\subsection{Relationships between $P_{n}^{B}(-1,1,1)$ and Euler number}

An alternating permutation on $[n]$ is defined as a permutation $\sigma=\sigma_{1} \sigma_{2} \cdots \sigma_{n} \in \mathfrak{S}_{n}$ such that

$$
\sigma_{1}>\sigma_{2}<\sigma_{3}>\sigma_{4}<\cdots \sigma_{n} .
$$

Denote by $\mathcal{E}_{n}$ the set of alternating permutations in $\mathfrak{S}_{n}$, and $E_{n}=\left|\mathcal{E}_{n}\right|$. It is well-known that $E_{n}$ is the Euler number and its exponential generating function [1] is given by

$$
\sum_{n \geqslant 0} E_{n} \frac{t^{n}}{n !}=\sec t+\tan t
$$

In particular, we have

$$
\sec t=\sum_{n \geqslant 0} E_{2 n} \frac{t^{2 n}}{(2 n) !} \quad \text { and } \quad \tan t=\sum_{n \geqslant 0} E_{2 n+1} \frac{t^{2 n+1}}{(2 n+1) !} .
$$

On substituting $x=-1, y=1$ and $z=1$ into Eq. (10), we have

$$
\sum_{n \geqslant 0} P_{n}^{B}(-1,1,1) \frac{t^{n}}{n !}=\frac{2 e^{2 t}}{1+e^{4 t}}
$$

Regarding the generating functions of the Euler number and $P_{n}^{B}(-1,1,1)$, we have

Theorem 9. For $n \geqslant 1$,

$$
\sum_{\sigma \in B_{n}}(-1)^{\operatorname{exc}_{B}(\sigma)}= \begin{cases}0, & \text { if } n \text { is odd } \\ (-1)^{\frac{n}{2}} 2^{n} E_{n}, & \text { if } n \text { is even } .\end{cases}
$$

Proof. Using the connection between hyperbolic function sech $t$ and the trigonometric functions sect, we have

$$
\sum_{n \geqslant 0} P_{n}^{B}(-1,1,1) \frac{t^{n}}{n !}=\frac{2 e^{2 t}}{1+e^{4 t}}=\operatorname{sech}(2 t)=\sec (2 i t)=\sum_{n \geqslant 0}(-1)^{n} 2^{2 n} E_{2 n} \frac{t^{2 n}}{(2 n) !} .
$$

The proof is completed by equating the coefficient of $\frac{t^{n}}{n !}$ on both sides.

Recall that the Eulerian polynomial of types $B$ is defined by $B_{n}(x)=\sum_{\pi \in B_{n}} x^{\operatorname{des}_{B}(\pi)}$, where $\operatorname{des}_{B}(\pi)=\left|\left\{i \in[0, n-1]: \pi_{i}>\pi_{i+1}\right\}\right|$ and $\pi_{0}=0$. By a combinatorial expansion of $B_{n}(x)$, Chow [10] showed that

$$
\sum_{\sigma \in B_{n}}(-1)^{\operatorname{des}_{B}(\sigma)}= \begin{cases}0, & \text { if } \mathrm{n} \text { is odd } \\ (-1)^{\frac{n}{2}} 2^{n} E_{n}, & \text { if } \mathrm{n} \text { is even }\end{cases}
$$

By the notice that the statistics $\operatorname{des}_{B}$ and $\operatorname{exc}_{B}$ are equi-distributed on $B_{n}$, this phenomenon is no coincidence. In what follows, we will give a combinatorial interpretation for Theorem 9 focusing on the statistic $\operatorname{exc}_{B}$. 
We begin with some definitions about alternating cycles. For $\sigma \in \mathfrak{S}_{n}$, an integer $i \in[n]$ is said to be a cycle peak (resp., cycle valley) of $\sigma$ if $\sigma^{-1}(i)<i>\sigma(i)$ (resp., $\left.\sigma^{-1}(i)>i<\sigma(i)\right)$. A cycle of the permutation $\sigma \in \mathfrak{S}_{n}$ is called an alternating cycle if every element in this cycle is either a cycle peak or a cycle valley, and the permutation $\sigma$ is called cycle-alternating if it is a product of alternating cycles. Denote by $\mathfrak{C}_{n}$ the set of cycle-alternating permutations in $\mathfrak{S}_{n}$.

It is easy to check that the length of an alternating cycle is always even. For a permutation in $\mathfrak{S}_{n}$ with $n$ odd, there exists at least one cycle of odd length, thus there is no cycle-alternating permutations in $\mathfrak{S}_{n}$. While for even $n$, the number of cycle-alternating permutations in $\mathfrak{S}_{n}$ equals the number of alternating permutations in $\mathfrak{S}_{n}$.

To see this, let $\sigma=\sigma_{1} \sigma_{2} \cdots \sigma_{n} \in \mathcal{E}_{n}$ with $\sigma_{m_{1}}<\sigma_{m_{2}}<\cdots<\sigma_{m_{k}}$ being its leftto-right maxima, and then set $C_{i}=\left(\sigma_{m_{i}}, \sigma_{m_{i}+1}, \ldots, \sigma_{m_{i+1}-1}\right)$ for $1 \leqslant i \leqslant k-1$ and $C_{k}=\left(\sigma_{m_{k}}, \ldots, \sigma_{n}\right)$. We define $\eta(\sigma)=C_{1} C_{2} \cdots C_{k}$ as the union of the $k$ disjoint cycles, and it is obvious that $\eta(\sigma)$ is a cycle-alternating permutation. For example, if $\sigma=31745286$, then its left-to-right maxima are $3,7,8$, and $\eta(\sigma)=(3,1)(7,4,5,2)(8,6) \in \mathfrak{C}_{8}$.

Lemma 10. Given a signed cycle $C$, let $|C|$ be the cycle obtained from $C$ by changing every negative element in it into positive. If the cycle $|C|$ is an alternating cycle, then we have $\operatorname{exc}_{B}(C)=\operatorname{exc}_{B}(|C|)$.

Proof. Suppose $|C|=\left(c_{1}, c_{2}, \ldots, c_{k}\right)$. If $C$ has $k$ negative elements $\overline{c_{i_{1}}}, \ldots, \overline{c_{i_{k}}}$, then we can generate such a cycle $C$ from $|C|$ by adding the minus sign from left to right to those $k$ negative elements in $C$.

Let $C_{j}(1 \leqslant j \leqslant k)$ be the cycle obtained from $|C|$ by attaching the minus sign to the elements $c_{i_{1}}, \ldots, c_{i_{j}}$, and set $C_{0}=|C|$. We will proceed by induction to show that $\operatorname{exc}_{B}\left(C_{j}\right)=\operatorname{exc}_{B}\left(C_{j-1}\right)$ for $1 \leqslant j \leqslant k$.

For $j=1$, if $c_{i_{1}}$ is a cycle peak, i.e., $c_{i_{1}-1}<c_{i_{1}}>c_{i_{1}+1}$, then $c_{i_{1}-1}>\overline{c_{i_{1}}}<c_{i_{1}+1}$; if $c_{i_{1}}$ is a cycle valley, i.e., $c_{i_{1}-1}>c_{i_{1}}<c_{i_{1}+1}$, then $c_{i_{1}-1}>\overline{c_{i_{1}}}<c_{i_{1}+1}$. It is easy to see that $\operatorname{exc}_{B}\left(C_{1}\right)=\operatorname{exc}_{B}(|C|)$.

Suppose that $\operatorname{exc}_{B}\left(C_{j}\right)=\operatorname{exc}_{B}\left(C_{j-1}\right)$ holds for all $j \leqslant m-1(2 \leqslant m \leqslant k)$. Since the cycle $C_{m}$ is constructed from the cycle $C_{m-1}$ by attaching the minus sign to $c_{i_{m}}$, it suffices to observe the difference between the excedance sets of cycles $C_{m-1}$ and $C_{m}$ after sign attachment of $c_{i_{m}}$. We continue our discussion according to the order of $c_{i_{m}}$ as follows:

- For a cycle peak $c_{i_{m}}$, we have $c_{i_{m}-1}<c_{i_{m}}>c_{i_{m}+1}$ in $C_{m-1}$ whereas $c_{i_{m}-1}>\overline{c_{i_{m}}}<$ $c_{i_{m}+1}$ in $C_{m}$ if $c_{i_{m}-1} \neq c_{i_{m-1}}$; otherwise, we have $\overline{c_{i_{m}-1}}<c_{i_{m}}>c_{i_{m}+1}$ in $C_{m-1}$ whereas $\overline{c_{i_{m}}-1}>\overline{c_{i_{m}}}<c_{i_{m}+1}$ in $C_{m}$;

- For a cycle valley $c_{i_{m}}$, we have $c_{i_{m}-1}>c_{i_{m}}<c_{i_{m}+1}$ in $C_{m-1}$ whereas $c_{i_{m}-1}>\overline{c_{i_{m}}}<$ $c_{i_{m}+1}$ in $C_{m}$ if $c_{i_{m}-1} \neq c_{i_{m-1}}$; otherwise, we have $\overline{c_{i_{m}-1}}<c_{i_{m}}<c_{i_{m}+1}$ in $C_{m-1}$ whereas $\overline{c_{i_{m}}-1}<\overline{c_{i_{m}}}<c_{i_{m}+1}$ in $C_{m}$.

Therefore, both of them imply that $\operatorname{exc}_{B}\left(C_{m}\right)=\operatorname{exc}_{B}\left(C_{m-1}\right)$, i.e., the statement is true for $j=m$. We complete the proof from $\operatorname{exc}_{B}(C)=\operatorname{exc}_{B}\left(C_{k}\right)=\operatorname{exc}_{B}\left(C_{0}\right)=\operatorname{exc}_{B}(|C|)$. 
Combinatorial Proof of Theorem 9. For a type $B$ permutation $\pi \in B_{n}$ with cycle decomposition $\pi=C_{1} \cdots C_{k}$, we proceed the proof by distinguishing the parity of $n$.

For odd $n$, there exists at least one cycle of odd length in $\pi$. Suppose that $C_{i_{1}}, \ldots, C_{i_{m}}$ are the odd cycles of $\pi$, and choose the odd cycle $C_{j}$ such that the cycle $\left|C_{j}\right|$ has the smallest minimum among all the minima of the cycles $\left|C_{i_{1}}\right|, \ldots,\left|C_{i_{m}}\right|$. Assume that $C_{j}=$ $\left(c_{1}, c_{2}, \ldots, c_{l}\right)$, and set $\overline{C_{j}}=\left(\overline{c_{1}}, \overline{c_{2}}, \ldots, \overline{c_{l}}\right)$, we define $\tau(\pi)=C_{1} \cdots C_{j-1} \overline{C_{j}} C_{j+1} \cdots C_{k}$. Since the only difference of the excedance sets between $\pi$ and $\tau(\pi)$ lies in the cycle $C_{j}$, while $\operatorname{exc}_{B}\left(C_{j}\right)=l-\operatorname{exc}_{B}\left(\overline{C_{j}}\right)$ and $(-1)^{\operatorname{exc}_{B}\left(C_{j}\right)}=-(-1)^{\operatorname{exc}_{B}\left(\overline{C_{j}}\right)}$ for odd $l$, this yields that $\operatorname{exc}_{B}(\pi)$ and $\operatorname{exc}_{B}(\tau(\pi))$ have different parity. Therefore, $\tau$ is the desired involution on $B_{n}$, and we complete the first part in Eq. (11).

For even $n$, it suffices to consider that all the cycles of $\pi=C_{1} \cdots C_{k}$ are of even length, otherwise we could make similar analysis as above when $\pi$ contains odd cycles.

If there exist some non-alternating cycles in $|\pi|=\left|C_{1}\right| \cdots\left|C_{k}\right|$, then we denote by $\left|C_{j}\right|=\left(\left|c_{1}\right|,\left|c_{2}\right|, \ldots,\left|c_{l}\right|\right)$ the non-alternating cycle which has the smallest minimum among all the minima of the non-alternating cycles of $|\pi|$. Let $\left|c_{i}\right|$ be the smallest element of $\left|C_{j}\right|$ such that it is neither a cycle peak nor a cycle valley. Define $\widehat{C_{j}}=$ $\left(c_{1}, \ldots, c_{i-1}, \overline{c_{i}}, c_{i+1}, \ldots, c_{l}\right)$ and we will show that $(-1)^{\operatorname{exc}_{B}\left(C_{j}\right)}=-(-1)^{\operatorname{exc}_{B}\left(\widehat{C_{j}}\right)}$.

We assume $c_{i}>0$ without loss of generality, and denote by $a=\left|c_{i-1}\right|, b=\left|c_{i}\right|$ and $c=\left|c_{i+1}\right|$ for simplicity. Suppose $a<b<c$ in the cycle $\left|C_{j}\right|$, it is easy to check:

i) If $C_{j}=(\ldots, a, b, c, \ldots)$, then $a<b<c$ in $C_{j}$ and $a>\bar{b}<c$ in $\widehat{C}_{j}$;

ii) If $C_{j}=(\ldots, \bar{a}, b, c, \ldots)$, then $\bar{a}<b<c$ in $C_{j}$ and $\bar{a}>\bar{b}<c$ in $\widehat{C_{j}}$;

iii) If $C_{j}=(\ldots, a, b, \bar{c}, \ldots)$, then $a<b>\bar{c}$ in $C_{j}$ and $a>\bar{b}>\bar{c}$ in $\widehat{C_{j}}$;

iv) If $C_{j}=(\ldots, \bar{a}, b, \bar{c}, \ldots)$, then $\bar{a}<b>\bar{c}$ in $C_{j}$ and $\bar{a}>\bar{b}>\bar{c}$ in $\widehat{C_{j}}$.

This implies that $(-1)^{\operatorname{exc}_{B}\left(C_{j}\right)}=-(-1)^{\operatorname{exc}_{B}\left(\widehat{C_{j}}\right)}$, and similar consideration could be made for the case $a>b>c$ in the cycle $\left|C_{j}\right|$.

We now consider the remaining situation when all the cycles of $|\pi|$ are alternating cycles. Since the cycles of $\pi$ are obtained from the cycles of $|\pi|$ by attaching minus signs for some elements, Lemma 10 shows that the type $B$ excedance number of each cycle $C \in \pi$ is the same as the corresponding alternating cycle $|C| \in|\pi|$. For an alternating cycle $C$ of length $2 l$, we have $\operatorname{exc}_{B}(C)=l$ and we can construct a signed cycle $C^{\prime}$ with $\left|C^{\prime}\right|=C$ in $2^{2 l}$ ways. The second part of Eq. (11) follows from the fact that the number of cycle-alternating permutations of even length $n$ is $E_{n}$, and each type $B$ permutation $\sigma$ with $|\sigma|$ being cycle-alternating can be constructed by attaching minus signs from $|\sigma|$ in $2^{n}$ ways. This completes the proof.

\subsection{Relationships between $P_{n}^{B}(-1,0,1)$ and Springer number}

A snake of type $B_{n}$ is an alternating type $B$ permutation $\sigma=\sigma_{1} \sigma_{2} \cdots \sigma_{n} \in B_{n}$ such that

$$
0<\sigma_{1}>\sigma_{2}<\sigma_{3}>\sigma_{4}<\cdots \sigma_{n}
$$

Arnol'd [2] proved that the number of snakes of type $B_{n}$ is equal to the $n$-th Springer number $S_{n}$, which was first introduced by Springer [14] in the study of irreducible root 
systems of type $B_{n}$. The generating function of $S_{n}$ is given by

$$
\sum_{n \geqslant 0} S_{n} \frac{t^{n}}{n !}=\frac{1}{\cos t-\sin t}
$$

On the other hand, setting $x=-1, y=0$ and $z=1$ into Eq. (10) gives

$$
\sum_{n \geqslant 0} P_{n}^{B}(-1,0,1) \frac{t^{n}}{n !}=\frac{2 e^{t}}{1+e^{4 t}}=\frac{\cosh t-\sinh t}{\cosh 2 t} .
$$

Therefore, we get the following theorem from their generating functions.

Theorem 11. For $n \geqslant 1$, we have

$$
\sum_{\sigma \in D_{n}^{B}}(-1)^{\operatorname{exc}_{B}(\sigma)}=(-1)^{\left\lfloor\frac{n+1}{2}\right\rfloor} S_{n} .
$$

Here we leave an open problem whether there exists a combinatorial interpretation of Theorem 11.

\section{Acknowledgments}

The author would like to thank the referee for many helpful suggestions and comments on a previous version of this paper. This work was supported by the National Science Foundation of China (\#11226301).

\section{References}

[1] D. André. Développement de sec $x$ et $\tan x$. C.R. Acad. Sci. Paris, 88:965-967, 1879.

[2] V. I. Arnol'd. The calculus of snakes and the combinatorics of Bernoulli, Euler, and Springer numbers of Coxeter groups. Uspekhi Mat. Nauk., 47:3-45, 1992. English translation in Russian Math. Surveys, 47:1-51, 1992.

[3] E. Bagno, A. Butman, and D. Garber. Statistics on the multi-colored permutation groups. Electron. J. Combin., 14 \#R24, 2007.

[4] E. Bagno, and D. Garber. On the excedance number of colored permutation groups. Sém. Lothar. Combin., 53 Art. B53f, 2006.

[5] F. Brenti. q-Eulerian polynomials arising from Coxeter groups. European J. Combin., 15:417-441, 1994.

[6] F. Brenti. A class of $q$-symmetric function arising from plethysm. J. Combin. Theory Ser. A, 91:137-170, 2000.

[7] R. Chapman. An involution on derangements. Discrete Math., 231:121-122, 2001.

[8] W. Y. C. Chen, R. L. Tang, and A. F. Y. Zhao. Derangement polynomials and excedances of type B. Electron. J. Combin., 16(2) \#R15, 2009. 
[9] C.-O. Chow, and I. M. Gessel. On the descent numbers and major indices for the hyperoctahedral group. Adv. in Appl. Math., 38:275-301, 2007.

[10] C.-O. Chow. On certain combinatorial expansions of the Eulerian polynomials. Adv. in Appl. Math., 41:133-157, 2008.

[11] C.-O. Chow. On derangement polynomials of type B. II. J. Combin. Theory Ser. A, 116:816-830, 2009.

[12] G. Ksavrelof, and J. Zeng. Two involutions for signed excedance numbers. Sém. Lothar. Combin., 49 Art. B49e, 2003.

[13] R. Mantaci, and F. Rakotondrajao. Exceedingly deranging!. Adv. in Appl. Math., 30:177-188, 2003.

[14] T. A. Springer. Remarks on a combinatorial problem. Nieuw Arch. Wisk., 19:30-36, 1971.

[15] R. P. Stanley. Enumerative Combinatorics, vol. 2, Cambridge University Press, 1997. 